\title{
A self adaptive new crossover operator to improve the efficiency of the genetic algorithm to find the shortest path
}

\author{
Mrinmoyee Chattoraj, Udaya Rani Vinayakamurthy \\ School of Computing \& IT, REVA University, Bengaluru, India
}

\begin{tabular}{l}
\hline \hline Article Info \\
\hline Article history: \\
Received Feb 15, 2021 \\
Revised May 29, 2021 \\
Accepted Jun 1, 2021 \\
\hline
\end{tabular}

\section{Keywords:}

Chromosome representation

Convergence

Genetic algorithm

Order crossover

Point based crossover

\begin{abstract}
Route planning is an important part of road network. To select an optimized route several factors such as flow of traffic, speed limits of road. are concerned. Total cost of such a network depends on the number of junctions between the source and the destination. Due to the growth of the nodes in the network it becomes a tough job to determine the exact path using deterministic algorithms so in such cases genetic algorithms (GA) plays a vital role to find the optimized route. Crossover is an important operator in genetic algorithm. The efficiency of the genetic algorithm is directly influenced by the time of a crossover operation. In this paper a new crossover operator closest-node pairing crossover (CNPC) is recommended which is explicitly designed to improve the performance of the genetic algorithm compared to other well-known crossover operators such as point based crossover and order crossover. The distance aspect of the network problem has been exploited in this crossover operator. This proposed technique gives a better result compared to the other crossover operator with the fitness value of 0.0048 . The CNPC operator gives better rate of convergence compared to the other crossover operators.
\end{abstract}

This is an open access article under the CC BY-SA license.

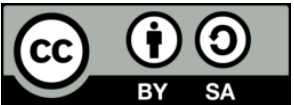

\section{Corresponding Author:}

Mrinmoyee Chattoraj

School of Computing \& IT

REVA University

Kattigenahalli, Yelahanka, Bengaluru, India

Email: mrinmoyee2005@gmail.com

\section{INTRODUCTION}

In the recent era, route optimization is gaining a lot of importance. There are various techniques to find the correct path. A lot of significance is given to genetic algorithms since it helps us to give an end-toend optimized solution. In case of the current road network as the rate of traffic increases, the service quality also decreases. In case of genetic algorithms from individual search space is generated where a respective individual gives a specific solution. genetic algorithms (GAs) which was developed by Holland in 1992, simulated Darwin's evolution theory through natural selection by a particular type of bio-inspired approach. According to this theory there is maximum chances for the survival of the fittest organism. In the search space, genetic algorithm will explore all the solutions and the optimal solution will be retained. All individuals of a particular solution are encoded in the form of chromosomes. The important genetic operators such as crossover and mutation are applied to the parent chromosome to achieve better solutions with more potential. Crossover operator recombines the offspring's and produces new chromosomes which are more enhanced than the parent chromosomes. To discover new states, mutation is often always needed, and it helps the genetic algorithm to escape local optima. These practises typically result in finding an optimal or nearoptimal global solution to a given problem [1], [2]. There are various types of crossover operators which are 
application dependent as well as application independent. A genetic algorithm's efficiency depends as to what kind of crossover operator used [3]-[5]. In this review, the main emphasis is on an important type of problems with combinatorial optimization whose solutions can be expressed with permutation. In this type of problem, the job is to arrange some objects in order to obtain the objective function where no duplicates are allowed. Some examples of these types of problems are Linear ordering problem, shortest path problem and travelling salesperson problem [6]. Permutation-based Gas are genetic algorithms that use path representation for chromosomes. Generally, for a set of integer's permutations is performed in order to encode a path. For permutation problems, this is the most general representation of chromosomes [7]. The path representation and suggested crossover and mutation operators unique to this representation have been used in a range of relevant studies on the application of GAs to permutation problems. In this analysis, we suggest a GA focused on permutation to answer problems of combinatorial optimization. The main attribute of this GA contains an improved crossover operator which improves the performance of the GA. In order to assess our crossover operator's performance and feasibility, we compare it with two well-known crossover operators Point Based Crossover and Order Crossover. The remaining part of the paper is arranged as follows: section 2, consists of a short description of GAs for problems of permutation-based combinatorial optimization. Section 3 describes the problem. Section 4, explains the methodology used in our proposed operator. Section 5, evaluates and discusses the results based on the results. Finally, conclusions are given in section 6.

\section{BACKGROUND}

Combinatorial optimization is a technique focused on finding an object (e.g. a graph) from a finite set of mathematical objects that significantly reduces or enhances a certain function. Variables are usually discrete in combinatorial optimization techniques. Permutation-based problems with combinatorial optimization are a major class of problems with combinatorial optimization whose solutions are defined as permutations. Finding the Shortest path is one of the combinatorial optimization problems where we try to minimize the total distance travelled as well as the time taken.

Inspired by Darwin's theory of evolution and natural selection, GAs are highly parallel search algorithms that develop a population of encoded candidate solutions (also called chromosomes) where each chromosome have a related fitness value and they undergo a set of genetic operations and finally new population is generated. There are various methods to represent chromosome for combinatorial optimisation problems [8]-[12]. In order to meet the requirement of the diverse need a variety of crossover and mutation operators have been developed.

In order to find an optimal solution for shortest path a lot of modification have been done on the crossover operator of the genetic algorithm to improve its efficiency. A crossover operator is represented in [13], [14], which generates a single crossover point, on the basis of cost comparison. This is a very simple approach with less difficulty but it is hard to achieve the optimum minimum cost of travel. Partially mapped crossover (PMX) was suggested in [15], [16]. This procedure selects a two-point crossover operator that samples the parent chromosome into three substrings and swaps the middle substring. Sequential Constructive Crossover Operator (SCX) [17], [18] produces an offspring from few parents using good edges based on their features that might be present in the arrangement of the parents to preserve the succession of nodes in the parent chromosomes. The order crossover (OX) suggested in [19]-[22] generates offspring by choosing a sub-tour from one parent and retaining the general order of bits of the other parent, which is also focused on the crossover operator with two points. In case of Cycle crossover operator (CX) proposed in [22]-[24] bits are taken from both parents in a circular order along with their position. This operator gives a good result but the drawback is that it gives the same offspring's with the same parents [25], [26].

However, it has been found in the described approaches certain nodes are reused over and over again so it is not feasible to attain diversity. The offspring acquired by crossover operators is identical to their parent allele and can thus does not achieve evolution. By minimising the replication of nodes, the suggested new crossover strategy proposed in this paper will overcome these limitations. In this paper we consider the "New York City Taxi and Limousine Commission" data set. The source and destination point of the taxi trip are considered as nodes and the shortest distance between each pair of nodes is calculated.

\section{METHODOLOGY}

Each feasible route for the path is represented by a chromosome. Randomly we create the initial population and the fitness function is the total distance of the route. For this Genetic algorithm we use Tournament Selection as the selection operator and Swap mutation as the mutation operator. We iterate it maximum number of times to reach the termination condition. We propose a crossover operator closest-node 
pairing crossover (CNPC) and compare it with existing crossover operators like Point Based Crossover and Order Crossover.

Pseudocode of closest node pairing crossover operator:

The steps involved in the crossover operator to obtain a child $i$ from a parent $i$ considers the distance between two genes.

a. Initially we select genes in a random manner from the parent chromosome and put it directly in the same location of the child chromosome.

b. In case the first gene of the child chromosome $\mathrm{i}$ is not assigned then we select randomly from the remaining chromosome of the unassigned genes from the parent $i$ and allocate it to the child.

c. For the remaining unassigned genes of the child chromosome $\mathrm{x}$ we follow the steps:

- Starting from left we find out the gene that is nearest to the assigned gene and assign it to the child $\mathrm{i}$ location.

- Similarly, we select the remaining unassigned gene from parent $\mathrm{i}$ which is close to the assigned gene in step a.

- This process is repeated till all the genes from parent $\mathrm{x}$ have been assigned in child $\mathrm{i}$.

d. We repeat the same process to create Child 2 from Parent 2 since the initial random selection creates a pair of parents at a time.

Figure 1 (a) illustrates the working process of the crossover operator for a network of eight nodes A, B, C, D, E, F, G and H which is shown in Figure 1 (b). On the arc we represent the distance between the nodes. The process begins by randomly selecting genes $\mathrm{E}, \mathrm{C}, \mathrm{H}$ and $\mathrm{F}$ from the Parent to the child and assigning it in the same chromosomal position. Since the first position of the child chromosome is not assigned so we randomly select any gene from the unassigned genes of the parent. In this example A, B, D and $\mathrm{G}$ are the unassigned genes so we had selected $\mathrm{G}$. For all other genes we select that gene which is closest to the gene on the left. In place of the third gene of the child chromosome we place D since it is nearest to gene $\mathrm{E}$. This process is repeated for all the unassigned genes in the parent chromosome. Thus, we obtain a child chromosome from a parent chromosome. This process is repeated to obtain child 2 for the parent.

In case of order crossover $(\mathrm{OX})$ to generate a feasible offspring both the parents are required. In the beginning we select a substring from Parent lin a random order. Then those genes selected from the first parent are deleted in the second parent and the remaining genes from the second parent are put in the child chromosome. Similarly, the second child is created by considering the first set of genes from Parent 2. Position based crossover (PBX) also requires both the parents to generate two offspring. In this case we randomly select a set of genes from the Parent 1 and transfer it to its children. It may or may not be a substring. The selected genes from Parent1 are deleted from Parent 2 and then the remaining genes from Parent 2 are transferred to the child. Figure 2 (a) illustrates order crossover whereas Figure 2 (b) illustrates position-based crossover for the same network shown in Figure 1.

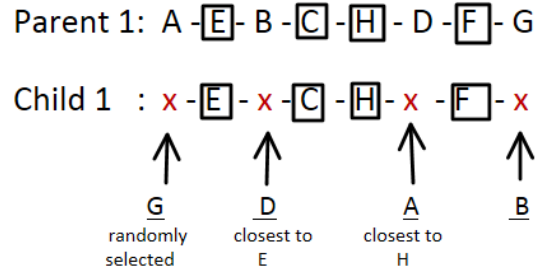

(a)

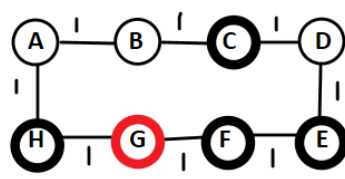

(b)

Figure 1. These figures are; (a) closestnode pairing croddover operator; (b) network diagram of 8 nodes

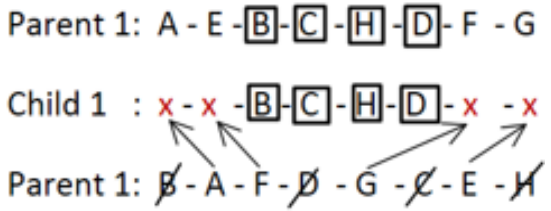

(a)

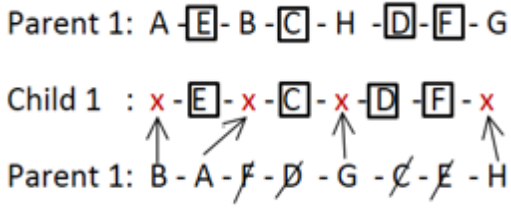

(b)

Figure 2. These figures are; (a) order crossover visual illustration; (b) position based crossover visual illustration 


\section{RESULTS AND DISCUSSION}

Genetic algorithm was used with the tournament size of 2, mutation rate $5 \%$ and termination condition of 10,000 iterations. We created 35 instances per crossover where the initial population are predetermined so that all the crossover operators have the same starting point. The following experiment was performed to compare our crossover operator with known crossover operators like OX and PBX. We change the population size and observe its effect on the distance of the route and its computation time. We also do a comparative study of the convergence rates of the three crossover operators. In our experiment we adjust our population size to $10,25,50,100$ and 200 . Hence a total of 5 (population size)* 3 (crossover operators) $* 30$ (instances) $=450$ test runs were conducted. We check the route distance and time taken for all the three crossover operators.

Table 1 shows the best, worst and average route distance obtained from the three crossover operators. It has been observed that from all the crossover operators the best route distance is 19086 metres. The worst and average route distances varies with the population size. In case of OX and PBX as the population size increases the average route distance decreases but in case of the new crossover operator the distance varies within a small range. Hence the population size does not make a major effect in the route distance.

Table 1. Best, worst and average route distance obtained from the three crossover, order crossover (OX),

\begin{tabular}{llccccc} 
position-based crossover (PBX) and the proposed crossover (CN \\
\cline { 2 - 7 } & \multicolumn{5}{c}{ POPULATION SIZE } \\
\hline \multirow{6}{*}{ OX } & Best & 10 & 25 & 50 & 100 & 200 \\
\hline \multirow{4}{*}{ PBX } & Worst & 21956 & 22367 & 22968 & 21539 & 20140 \\
& Average & 21530 & 20476 & 20498 & 19843 & 19337 \\
& Best & 19086 & 19086 & 19086 & 19086 & 19086 \\
& Worst & 22100 & 22050 & 22050 & 22680 & 21045 \\
CNPC & Average & 20775 & 20634 & 20173 & 19996 & 19570 \\
& Best & 19086 & 19086 & 19086 & 19086 & 19086 \\
& Worst & 19590 & 19990 & 19600 & 19600 & 19600 \\
& Average & 19401 & 19420 & 19359 & 19302 & 19265 \\
\hline
\end{tabular}

Figure 3 shows a comparison of the average route distance of the three crossover operators with respect to the population size and it has been observed that the route distance slightly decreases with respect to the increase in population size. Figure 4 shows a comparison of the average computation time of the three crossover operators with respect to the population size and it has been observed that the population size has very little effect on the computation time.

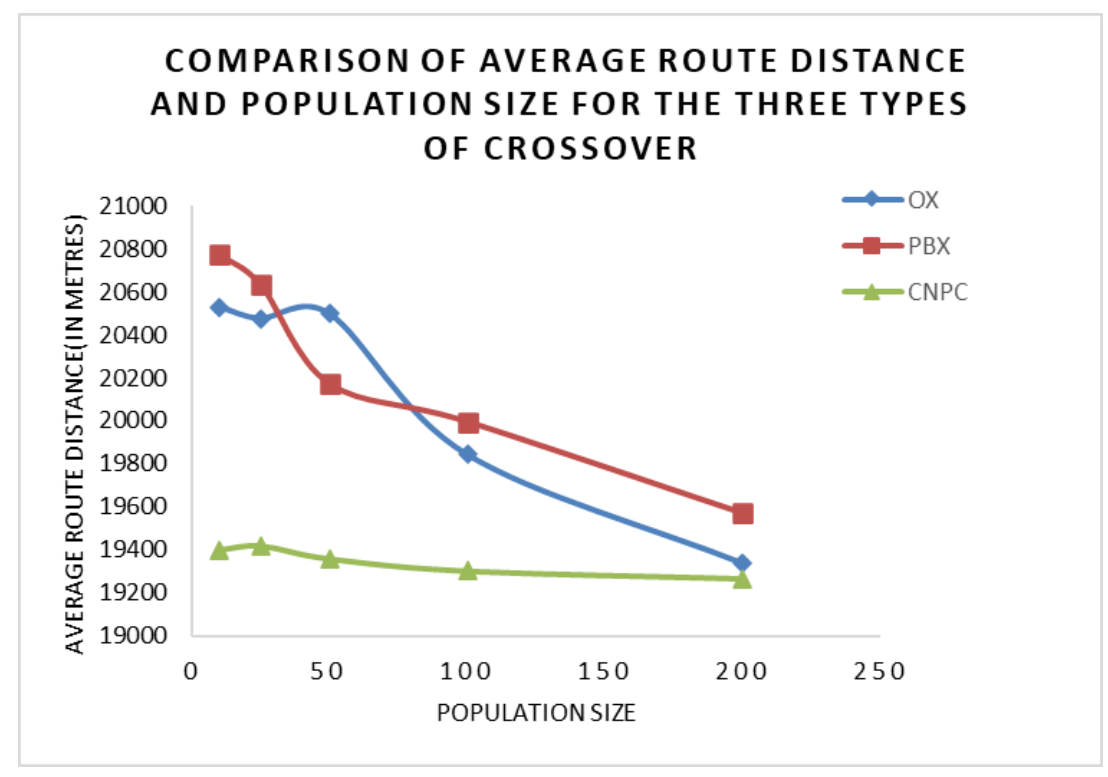

Figure 3. Comparison of average route distance and population size for the three types of crossovers 


\section{COMPARISON OF AVERAGE COMPUTATION TIME FOR THE CROSSOVER OPERATORS}

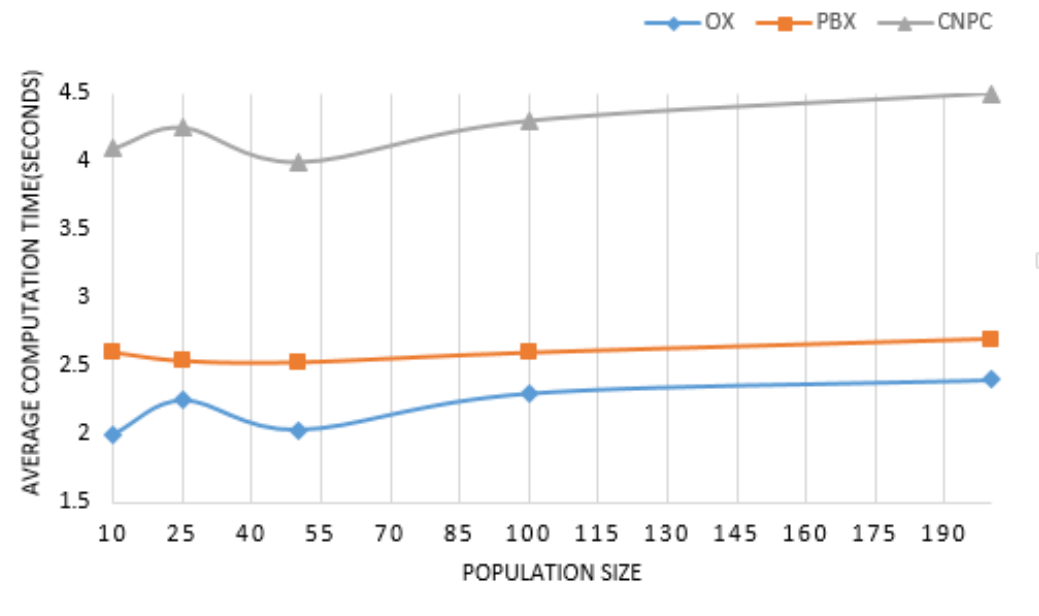

Figure 4. Comparison of average computation time with respect to population size

In genetic algorithm, we consider that the solution has converged only when the same offspring are generated. Solution convergence is good when we require less number of iterations. Here for population size 10 we perform the convergence experiment. The route and its distance are calculated for 500 iterations with 90 test runs ( 3 crossover operators*30 instances). In Figure 5 a comparative study of average route distance has been calculated for 500 iterations interval. It has been observed that the slowest convergence rate is of the OX crossover operator whereas CNPC outperforms the convergence rate of the OX and PBX operators.

According to the data it has been observed that the crossover operators OX and PBX converges after obtaining 10000 iterations but our proposed crossover operator CNPC makes significant changes at an earlier state of only 1500 iterations. Thus, we can say that in comparison to another crossover operators the proposed operator converges within a maximum range of 2000 iterations.

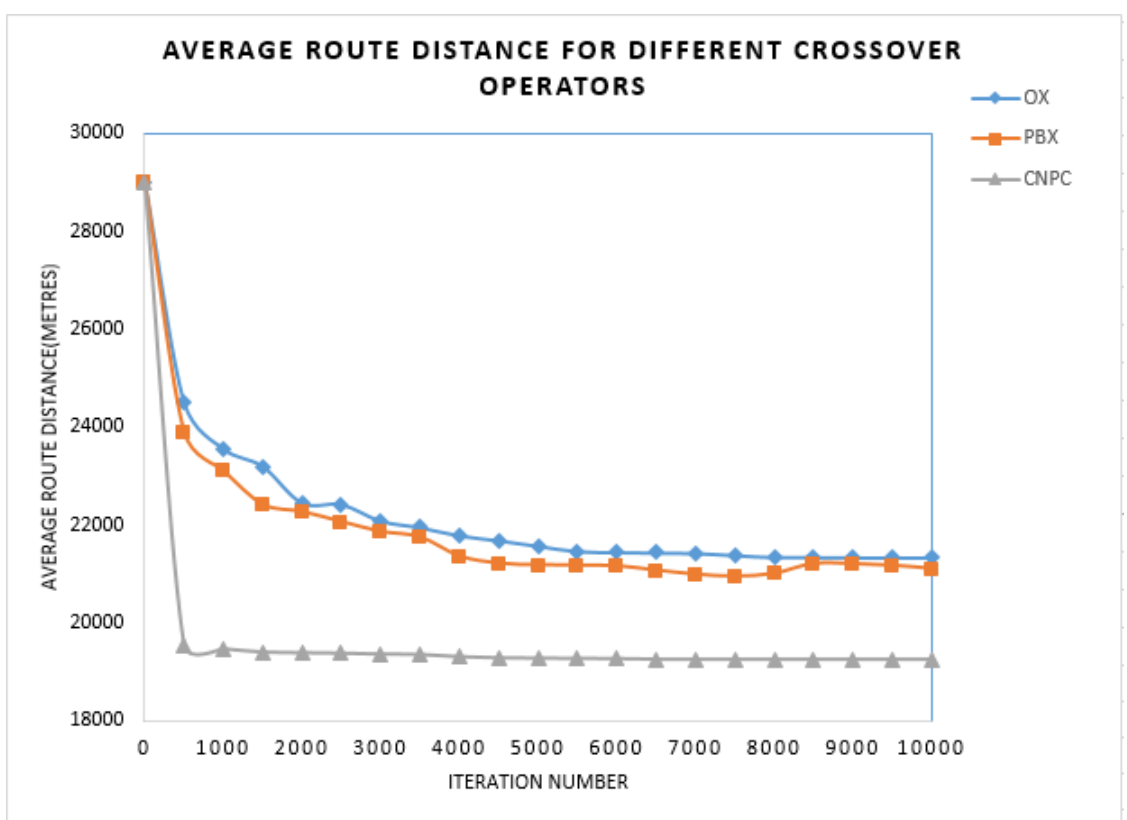

Figure 5. Comparison of average route distance with respect to iteration number for different crossover operators 


\section{CONCLUSION}

In order to find the shortest route using genetic algorithm we propose a new crossover operator closest node pairing crossover (CNPC) whose overall performance is better than OX and PBX which are the crossover operators which is very common. It has been observed that the performance of crossover operators mainly OX and PBX depends mostly on the population size while the CNPC operator is not much depended on the population size. The convergence rate of the CNPC operator is also faster as compared to the other crossover operators.

\section{REFERENCES}

[1] A. E. Eiben and J. E. Smith, Introduction to evolutionary computing, Berlin, Heidelberg: Springer, 2015.

[2] M. Chattoraj and V. U. Rani, "Road traffic network solution in Matlab using soft computing," 2017 International Conference on Smart Technologies for Smart Nation (SmartTechCon), 2017, pp. 1434-1437, doi: 10.1109/SmartTechCon.2017.8358601.

[3] A. El Beqal, B. Benhala, and I. Zorkani, "A genetic algorithm for the optimal design of a multistage amplifier," International Journal of Electrical and Computer Engineering, vol. 10, no. 1, pp. 129-138, 2020, doi: 10.11591/ijece.v10i1.pp129-138.

[4] A. J. Umbarkar and P. D. Sheth, "Crossover operators in genetic algorithms: A review," ICTACT journal on soft computing, vol. 6, no. 1, 2015, doi: 10.21917/ijsc.2015.0150.

[5] R. Pinho and F. Saraiva, "A comparison of crossover operators in genetic algorithms for switch allocation problem in power distribution systems," 2020 IEEE Congress on Evolutionary Computation (CEC), 2020, pp. 1-8, doi: 10.1109/CEC48606.2020.9185795.

[6] C. H. Papadimitriou and K. Steiglitz, Combinatorial optimization: Algorithms and complexity, Hawaii: Courier Corporation, 1998.

[7] K. L. Du and M. N. S. Swamy, "Search and optimization by metaheuristics," Techniques and Algorithms Inspired by Nature; Birkhauser: Basel, Switzerland, 2016.

[8] G. Pavai and T. V. Geetha, "A survey on crossover operators," ACM Computing Surveys (CSUR), vol. 49, no. 4, pp. 1-43, 2016, doi: 10.1145/3009966.

[9] L. Manzoni, L. Mariot, and E. Tuba, "Balanced crossover operators in genetic algorithms," Swarm and Evolutionary Computation, vol. 54, 2020, doi: 10.1016/j.swevo.2020.100646.

[10] P. Larranaga, C. M. H. Kuijpers, R. H. Murga, I. Inza, and S. Dizdarevic, "Genetic algorithms for the travelling salesman problem: A review of representations and operators," Artificial Intelligence Review, vol. 13, no. 2, pp. 129-170, 1999, doi: 10.1023/A:1006529012972.

[11] A. Turky, N. R. Sabar, S. Dunstall, and A. Song, "Hyper-heuristic local search for combinatorial optimisation problems," Knowledge-Based Systems, vol 205, 2020, doi: 10.1016/j.knosys.2020.106264.

[12] S. Masrom, M. Mohamad, S. M. Hatim, N. Baharun, N. Omar, and A. S. A. Rahman, "Different mutation and crossover set of genetic programming in an automated machine learning," IAES International Journal of Artificial Intelligence, vol. 9, no. 3, pp. 402-408, 2020, doi: 10.11591/ijai.v9.i3.pp402-408.

[13] S. Akter, N. Nahar, M. S. Hossain, and K. Andersson, "A new crossover technique to improve genetic algorithm and its application to TSP," 2019 International Conference on Electrical, Computer and Communication Engineering (ECCE), 2019, pp. 1-6, doi: 10.1109/ECACE.2019.8679367.

[14] R. B. Abduljabbar, O. K. Hamid, and N. J. Alhyani, "Features of genetic algorithm for plain text encryption," International Journal of Electrical and Computer Engineering (IJECE), vol. 11, no. 1, pp. 434-441, 2021, doi: 10.11591/ijece.v11i1.pp434-441.

[15] A. Hussain, Y. S. Muhammad, and M. N. Sajid, "A simulated study of genetic algorithm with a new crossover operator using traveling salesman problem," Punjab University Journal of Mathematics, vol. 51, no. 5, 2020.

[16] N. Attarmoghaddam, K. F. Li, and A. Kanan, "FPGA implementation of crossover module of genetic algorithm," Information, vol. 10, no. 6, 2019, doi: 10.3390/info10060184.

[17] Z. H. Ahmed, "Adaptive sequential constructive crossover operator in a genetic algorithm for solving the traveling salesman problem," (IJACSA) International Journal of Advanced Computer Science and Applications, vol. 11, no. 2, 593-605, 2020

[18] A. N. Singh, J. Mrudula, R. Pandey, and S. Das, "A Comparative Study of Four Genetic Algorithm-Based Crossover Operators for Solving Travelling Salesman Problem," Intelligent Algorithms for Analysis and Control of Dynamical Systems, 2021, pp. 33-40, doi: 10.1007/978-981-15-8045-1_4.

[19] A. Arram and M. Ayob, "A novel multi-parent order crossover in genetic algorithm for combinatorial optimization problems," Computers \& Industrial Engineering, vol. 133, pp. 267-274, 2019, doi: 10.1016/j.cie.2019.05.012.

[20] B. Kiraz, A. A. Bidgoli, H. E.-Komleh, and S. Rahnamayan, "A novel collective crossover operator for genetic algorithms," 2020 IEEE International Conference on Systems, Man, and Cybernetics (SMC), 2020, pp. 4204-4209, doi: 10.1109/SMC42975.2020.9282841.

[21] M. Y. Orong, A. M. Sison, and R. P. Medina, "A new crossover mechanism for genetic algorithm with rank-based selection method," 2018 5th International Conference on Business and Industrial Research (ICBIR), 2018, pp. 83-88, doi: 10.1109/ICBIR.2018.8391171. 
[22] J. A. Cano, P. C. Achedad, E. A. Campo, and A. A. C. Espinal, "Solving the order batching and sequencing problem with multiple pickers: A grouped genetic algorithm," International Journal of Electrical and Computer Engineering (IJECE), vol. 11, no. 3, pp. 2516-2524, 2021, doi: 10.11591/ijece.v11i3.pp2516-2524.

[23] V. Singh, L. Ganapathy, and A. K. Pundir, "An improved genetic algorithm for solving multi depot vehicle routing problem," International Journal of Information Systems and Supply Chain Management (IJISSCM), vol. 12, no. 4, pp. 1-26, 2019.

[24] M. H. Hassan, M. A. Jubair, S. A. Mostafa, H. Kamaludin, A. Mustapha, M. F. M. Fudzee, and H. Mahdin, "A general framework of genetic multi-agent routing protocol for improving the performance of MANET environment," IAES International Journal of Artificial Intelligence (IJ-AI), vol. 9, no. 2, pp. 310-316, 2020, doi: 10.11591/ijai.v9.i2.pp310-316.

[25] K. V. Sheelavathy and V. U. Rani, "Detection and classificaiton of malware using progressive principal component and attension based deep neural network," International Journal of Grid and Distributed Computing, vol. 13, no. 1, pp. 2332-2347, 2020.

[26] R. R. Savant, S. S. Nayak, S. Azam, and Udayarani "Driver safety system using machine learning," The Mattingley Publishing Co., Inc, vol. 83. no. May-June, pp. 4999-5003, 2020.

\section{BIOGRAPHIES OF AUTHORS}

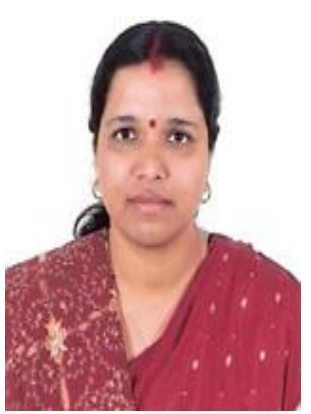

Mrinmoyee Chattoraj is a Research Scholar in "School of Computing \& Information Technology", Reva University, Bangalore. She has 15yrs of teaching experience. Currently she is working as an Assistant Professor St. Joseph's College, Bangalore. Her research interests are Computer Network, Machine Learning and Genetic Programming.

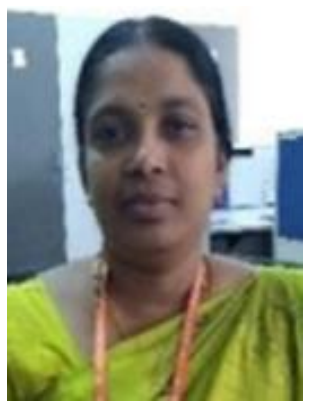

Dr. Udaya Rani Vinayakamurthy received her PhD from Mother Teresa University, Tamil Nadu, India in 2014. Since 2011 she is working as a Sr. Associate Professor in Reva University, Bangalore. She has approx 19yrs of experience and published above 20 papers in National and International Journals. Her research areas include Data Mining and Warehousing, Machine Learning, Big Data and Genetic Programming. 